\title{
вмJ Global Health Impact of a free care policy on the utilisation of health services during an Ebola outbreak in the Democratic Republic of Congo: an interrupted time- series analysis
}

To cite: Hung YW, Law MR, Cheng $L$, et al. Impact of a free care policy on the utilisation of health services during an Ebola outbreak in the Democratic Republic of Congo: an interrupted time-series analysis. BMJ Global Health 2020;5:e002119. doi:10.1136/ bmjgh-2019-002119

Handling editor Valery Ridde

- Additional material is published online only. To view please visit the journal online (http://dx.doi.org/10.1136/ bmjgh-2019-002119).

Received 28 0ctober 2019 Revised 8 May 2020 Accepted 9 May 2020

Check for updates

(C) Author(s) (or their employer(s)) 2020. Re-use permitted under CC BY-NC. No commercial re-use. See rights and permissions. Published by BMJ.

For numbered affiliations see end of article.

Correspondence to Dr Karen A Grépin; kgrepin@hku.hk

\section{ABSTRACT}

Background During past outbreaks of Ebola virus disease (EVD) and other infectious diseases, health service utilisation declined among the general public, delaying health seeking behaviour and affecting population health. From May to July 2018, the Democratic Republic of Congo experienced an outbreak of EVD in Equateur province. The Ministry of Public Health introduced a free care policy (FCP) in both affected and neighbouring health zones. We evaluated the impact of this policy on health service utilisation.

Methods Using monthly data from the national Health Management Information System from January 2017 to January 2019, we examined rates of the use of nine health services at primary health facilities: total visits; first and fourth antenatal care visits; institutional deliveries; postnatal care visits; diphtheria, pertussis and tetanus (DTP) vaccinations and visits for uncomplicated malaria, pneumonia and diarrhoea. We used controlled interrupted time series analysis with a mixed effects model to estimate changes in the rates of services use during the policy (June-September 2018) and afterwards.

Findings Overall, use of most services increased compared to control health zones, including EVD affected areas. Total visits and visits for pneumonia and diarrhoea initially increased more than two-fold relative to the control areas $(p<0.001)$, while institutional deliveries and first antenatal care increased between $20 \%$ and $50 \%(p<0.01)$. Visits for DTP, fourth antenatal care visits and postnatal care visits were not significantly affected. During the FCP period, visit rates followed a downward trend. Most increases did not persist after the policy ended. Interpretation The FCP was effective at rapidly increasing the use of some health services both EVD affected and not affected health zones, but this effect was not sustained post FCP. Such policies may mitigate the adverse impact of infectious disease outbreaks on population health

\section{Key questions}

What is already known?

- Evidence from previous outbreaks of Ebola virus disease (EVD) and other infectious diseases suggests that the use of primary health services usually declines among the general public, which can lead to important declines in population health.

- To our knowledge, there have been no evaluations of policies or strategies implemented to mitigate the impact of EVD outbreaks on the use of health services in any international context.

- The introduction of user fee exemption or other free care policies (FCPS) to incentivise health service utilisation have been evaluated in a number of SubSaharan African countries contexts, however, none have been evaluated in the context of an outbreak of EVD or other infectious diseases.

What are the new findings?

- Our findings provide strong evidence that utilisation rates of many primary health services increased with the implementation of the FCP, although most increases were not sustained after the FCP ended.

- Total visits and treatments for pneumonia and diarrhoea saw the largest increases while some services, such as those involving needles and blood, were not affected by the FCP.

What do the new findings imply?

- The available scientific evidence suggests that FCPs may be an effective strategy to mitigate the impact of EVD outbreaks on the use of health services among the general public, even in resource poor settings.

- Further research is needed to understand how such policies can be better implemented and additional strategies should also be explored.

- Routine health information system data can be a useful tool to study the impact of FCPs and othe short-term policies in low income country settings. 


\section{INTRODUCTION}

Widespread disruption to health systems has been observed during previous major outbreaks of infectious diseases. ${ }^{12}$ For example, during the 2014-2016 outbreak of Ebola virus disease (EVD) in West Africa, the use of health services greatly declined in heavily affected countries. ${ }^{34}$ Communities were fearful and lacked trust in the health system, which not only impeded response efforts, but also deterred health seeking behaviour. ${ }^{56}$ Overall use of health services decreased by $18 \%$ during the peak of the outbreak. ${ }^{7}$ Reductions were seen for maternal and child health services, ${ }^{189}$ as well as treatments for priority diseases such as malaria ${ }^{1011}$ and HIV. ${ }^{12}{ }^{13}$ It has been estimated that reductions in the use of health services led to mortality increases similar in magnitude to those directly attributable to EVD. ${ }^{8914}$ Moreover, studies have shown that patterns of health seeking behaviour were disrupted for months after the outbreak. ${ }^{19}$

As a result, implementing policies to mitigate these impacts should be a priority. In Africa, user fee exemption policies or other free care policies (FCPs) have been a popular approach to incentivise health service utilisation, especially in the use of maternal and child healthcare services. However, studies have shown mixed evidence with regards to their effectiveness in various contexts. ${ }^{15-18}$ Weak study designs, many of which lacked an adequate control group, may partially explain the mixed evidence. ${ }^{1618}$ An evaluation of a previous FCP introduced in the Democratic Republic of Congo (DRC) in 2008, unrelated to an EVD outbreak, also demonstrated mixed results and the programme was not sustained. ${ }^{19}$ While FCPs have been implemented in previous EVD outbreaks in DRC, to date, there have been no evaluations of their impact, where in addition to the usual concerns, additional challenges may further limit the effectiveness of such policies.

FCPs could provide benefits in two ways during an outbreak. First, it could encourage early identification and treatment of the disease itself-a factor that is believed to be a key predictor of Ebola survivorship. Second, it could help increase or maintain the use of other beneficial health services among the general population. Based on the intervention theory of health user fee exemption policies developed by Robert et al, a FCP should allow households to obtain health services that were previously unaffordable. ${ }^{20}$ However, in the context of an EVD outbreak, fear of infection or a lack of trust in the health system may limit the demand for these services.

In the 2018 EVD outbreak in the Equateur province of DRC, the Ministry of Public Health quickly implemented a temporary FCP in the three EVD affected health zones as well as in four neighbouring health zones, primarily motivated by the first of the benefits described above. ${ }^{21}$ The implementation was supported by the ongoing Health System Strengthening for Better Maternal and Child Health Results Project funded by the World Bank. ${ }^{21}$ In this paper, we evaluate the effect of the FCP on the use of health services at primary health centres (PHCs) in Equateur province using routinely collected administrative data.

\section{METHODS \\ Context}

The DRC is among the largest and most populous countries in Africa and also has some of the worst health indicators in the region. ${ }^{22}$ The health system is highly decentralised and is challenged with very low levels of funding. ${ }^{23}$ To compensate, the health system relies heavily on user fees for financing. However, as over $60 \%$ of the population live in poverty, user fees represent a major barrier to health service utilisation. ${ }^{23} 24$ The DRC has 26 provinces which are subdivided into 516 health zones. Each health zone is further subdivided into health areas, each of which is equipped with health centres to provide primary health services. $^{23}$

In 2018, the DRC experienced two separate EVD outbreaks: the first happened in Equateur province, followed by a second in the eastern region of the country a few months later. ${ }^{25}$ The first, which was declared on May 8, initially began in the Ikoko-Impenge health area in the Bikoro health zone, where two cases of fever were confirmed to be EVD and 17 community deaths had been reported. ${ }^{26}$ By May 14, a total of 41 cases and 20 deaths were reported, including probable cases from the Iboko and Wangata health zones, a distance of nearly $150 \mathrm{~km}$, raising concerns of widespread transmission. ${ }^{27}$ In response to the outbreak, the DRC Ministry of Public Health, in partnership with the WHO, established a social awareness campaign and delivered personal protective equipment to the region by May 11. On May 21, vaccination campaigns were launched targeting front-line health workers, individuals exposed to confirmed EVD cases and contacts of these individuals. ${ }^{27}$ Additionally, to encourage people at risk to seek medical care and improve surveillance in the community, the Ministry of Public Health implemented a temporary FCP in the health areas affected by the EVD epidemic as well as in nearby health zones, beginning in June $2018 .^{21}$ The outbreak infected a total of 54 people and led to 33 deaths, including two health workers, before being declared over on July 24 , with cases remained localised to the three health zones. ${ }^{28}$

\section{Study setting}

We conducted a retrospective, controlled interrupted time-series (ITS) study using monthly data to estimate changes in the level and trend in the rate of health service utilisation between January 2017 and January 2019 at PHCs during the Equateur province outbreak. Controlled ITS is a very strong quasi-experimental study design that can be used with routinely collected health system data. ${ }^{29}$ Within Equateur province, cases of EVD were reported in 3 of the 18 health zones: Bikoro (rural), Iboko (rural) and Wangata (urban). Along with these affected zones, four neighbouring health zones (Bolenge, Ingende, Ntondo and Mbandaka) also received the FCP, 


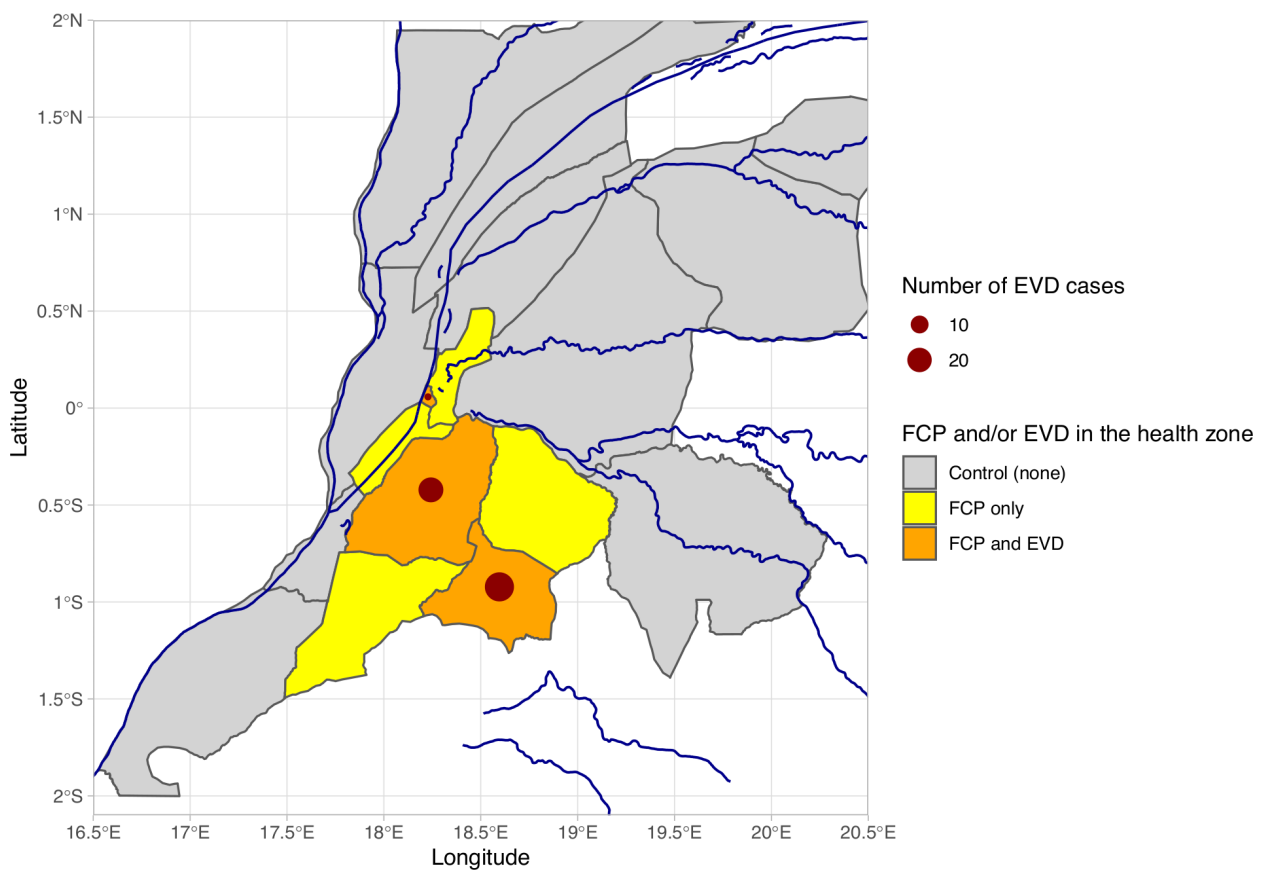

Figure 1 Map of health zones by status of free care policy (FCP) and Ebola virus disease (EVD) (probable and confirmed) cases in Equateur province, Demographic Republic of Congo, 5 April-24 July 2018.

which was in effect between June and September 2018 (figure 1). FCP covered consultations and medications for EVD and other health conditions in the targeted areas. Payments were made from the government to public health facilities (health centres and hospitals) to support the health workers and the maintenance of the facilities, using an existing payment platform that had previously been established to support a results-based financing programme in the area. The government also distributed medicines covered by the FCP to public facilities. ${ }^{21}$ We confirmed the enactment and implementation dates of the FCP with both provincial health administrators and local healthcare workers in Equateur province.

\section{Data source and outcomes}

We extracted monthly data from the Health Management Information System (HMIS), an national electronic data collection system based on the District Health Information System 2 (DHIS2) platform. ${ }^{30}$ Data in this system are input from health facilities' monthly health service use reports at district health offices. Significant efforts have been launched in the DRC to improve the quality of HMIS data, including continual quality assessment activities at both the health zone and facility levels and incentives for report submission and completion. ${ }^{30} 31$ HMIS data have been used to retroactively evaluate the impact of the West African EVD outbreak on health service utilisation, ${ }^{189}$ and to evaluate the impact of other policies in other low-income and middle-income countries contexts using ITS analysis. ${ }^{32} 33$

For each PHC, we extracted the number of visits for the following health services:

1. Overall: (1) total clinic visits.
2. Common infectious diseases: (2) uncomplicated pneumonia cases diagnosed; (3) uncomplicated diarrhoea cases diagnosed; and (4) uncomplicated malaria cases diagnosed.

3. Maternal health: (5) institutional deliveries; (6) first and (7) fourth antenatal care visits; (8) postnatal care visits within 6 days of birth.

4. Vaccination: (9) first doses of diphtheria-tetanuspertussis vaccine (DTP) administered.

Each health facility reported each of the indicators on a monthly basis. To enable comparisons between health zones, monthly counts were modelled as per-capita monthly rates using the estimated catchment populations for each facility reported in the HMIS.

These indicators were selected as they represent the majority of health services delivered in PHCs $(79 \%$ of total visits) and had the highest level of data completeness. Although routine immunisations and malaria rapid diagnostic testing had been curtailed during the outbreak due to EVD transmission concerns, we included these indicators to monitor the overall use of health services in the general population in the context of the EVD outbreak. The research protocol was approved by the Ethics Committees at Wilfrid Laurier University (Canada) and Kinshasa School of Public Health (DRC).

\section{Statistical analyses}

We tested the following hypotheses: (1) Was the FCP associated with significant changes in health services utilisation at PHCs in both EVD affected and non-EVD affected health zones? If so, what were the magnitude of these changes? (2) Were the changes in health service utilisation sustained throughout the period of the FCP 
implementation and afterwards? (3) Were there any differential effects of the FCP in EVD vs non-EVD health zones? (4) Were some health services more affected by the FCP than others?

Our analysis included the PHCs in two intervention groups that received the FCP: three EVD affected health zones (EVD and FCP), as well as in the four neighbouring health zones that received the FCP but were not directly affected by EVD (FCP only). PHCs in the remaining health zones within Equateur province were included as the control group (neither). We fit our models using a two-level mixed-effects negative binomial model to adjust for the clustering of observations from the same health centres over time, and to correct for over-dispersion. All of our models included random intercepts for clinic in order to account for heterogeneity of clinic visit volumes, and an autoregressive structure of one period to account for potential correlation between observations over time. We defined three time periods: pre-intervention (January 2017-March 2018), intervention (June-September 2018) and post-intervention (October 2018-January 2019) based on the timing of the FCP. We excluded the first 2 months of the outbreak (April and May 2018) ${ }^{28}$ from our analysis, as the time period between the onset of the outbreak and the implementation of the FCP was too short to independently test for time trends. Each indicator was analysed for level and slope over time, changes in immediate (level) and gradual (slope) in the intervention and post-intervention period, and the interaction of these changes with the different study groups (EVD and FCP, FCP only, neither). PHCs were excluded from each analysis if data were missing for two or more consecutive months in either the pre-FCP (October 2017 and March 2018), or during EVD and FCP (April and September 2018) periods. Thus, we excluded PHCs from one health zone (Makanza) that had neither EVD nor FCP due to lack of consistent reporting. We identified outliers and excluded a PHC for a specific indicator if their reported data exceeded eight SD from the mean time trend $(<0.5 \%$ of the sample). Due to this rule about missing data, number of PHCs included in each study group varied by indicator. The samples of PHCs for each indicator is included in online supplementary table sla and s1b. We then conducted separate controlled ITS analysis for each of the nine indicators. Missing data were accounted for using standard maximum likelihood estimation in the mixed-effects models.

Additionally, in order to estimate the absolute change of each outcome compared to the counterfactual estimate without the FCP, we used the non-linear (exponential) combination of estimate parameters from the two-level mixed-effects negative binomial model and applied bootstrapping method to construct confidence intervals around the predicted absolute changes in outcome. We simulated data based on the estimates with normally distributed error using bootstrap statistics with 10000 resamples within each group. All analyses were conducted using SAS V.9.4. ${ }^{34}$

\section{Patient and public involvement}

This research was done without patient involvement. Patients were not invited to comment on the study design and were not consulted to develop patient relevant outcomes or interpret the results. Patients were not invited to contribute to the writing or editing of this document for readability or accuracy.

\section{RESULTS}

As shown in table 1, we found that the FCP was associated with changes in the utilisation of many types of services, majority were similar in both the health zones with EVD and in neighbouring health zones with FCP only.

\section{Overall}

Relative to control health zones, rates of total clinic visits increased substantially in FCP health zones following the start of the EVD outbreak and we see similar increases in both the EVD and FCP health zones and the FCP-only health zones. As shown in figure 2, visit rates increased more than twofold in EVD and FCP health zones (incidence rate ratio (IRR): $2.7,95 \% \mathrm{CI}: 2.2-3.2, \mathrm{p}<0.001$ ) and FCP-only health zones (IRR: 2.7, 95\% CI: 2.2-3.1, $\mathrm{p}<0.001)$. However, this large increase in visit rates was not maintained over the implementation period (EVD and FCP: IRR: 0.91, 95\% CI: 0.86-0.97, $\mathrm{p}=0.003$; FCP only: IRR: $0.90,95 \%$ CI: $0.85-0.95, \mathrm{p}<0.001)$. Following the end of the FCP, the level of total clinic visits dropped by $21 \%$ in EVD and FCP health zones (95\% CI: $0.65-0.96$, $\mathrm{p}=0.02$ ) and by $18 \%$ in FCP-only health zones (95\% CI: $0.68-1.0, \mathrm{p}=0.05$ ) compared to the FCP period, with no significant change in trend after the FCP ended (EVD and FCP: IRR: 1.0, 95\% CI: $0.97-1.1, \mathrm{p}=0.26$; FCP only: IRR: $1.1,95 \%$ CI: $0.98-1.2, \mathrm{p}=0.12$ ).

\section{Common infectious diseases}

Similar to overall clinic visits, the FCP was associated with a large increase in visits for pneumonia and diarrhoea, and to a smaller extent for malaria visits. Figure 3 shows the model results for the rate of clinic visits for pneumonia. Compared to facilities in control health zones, clinic visits for pneumonia doubled at the beginning of FCP in EVD and FCP health zones (IRR: 2.1, 95\% CI: 1.5-2.9, $\mathrm{p}<0.001)$ and quadrupled in FCP-only health zones (IRR: 4.1, 95\% CI: 2.9-5.9, p<0.001). During the implementation period, visits for pneumonia in EVD and FCP health zones had no significant change (IRR: 1.0, 95\% CI: $0.90-1.1, \mathrm{p}=0.94)$ while the rate decreased over time in FCP-only health zones (IRR: 0.79, 95\% CI: $0.71-$ $0.87, \mathrm{p}<0.001)$. Following the end of the FCP, the level of pneumonia visits decreased by $42 \%$ in EVD and FCP health zones $(p=0.001)$, with no significant difference in the trend in subsequent months (IRR: 1.0, 95\% CI: $0.89-1.2, \mathrm{p}=0.75)$. The $23 \%$ decrease in FCP-only health zones was not statistically significant (IRR: $0.77,95 \% \mathrm{CI}$ : $0.54-1.1, \mathrm{p}=0.16)$ and the trend reversed after the end of the policy (IRR: $1.3,95 \%$ CI: $1.1-1.5, \mathrm{p}<0.001$ ). Changes in the levels and trends of visits for diarrhoea following 


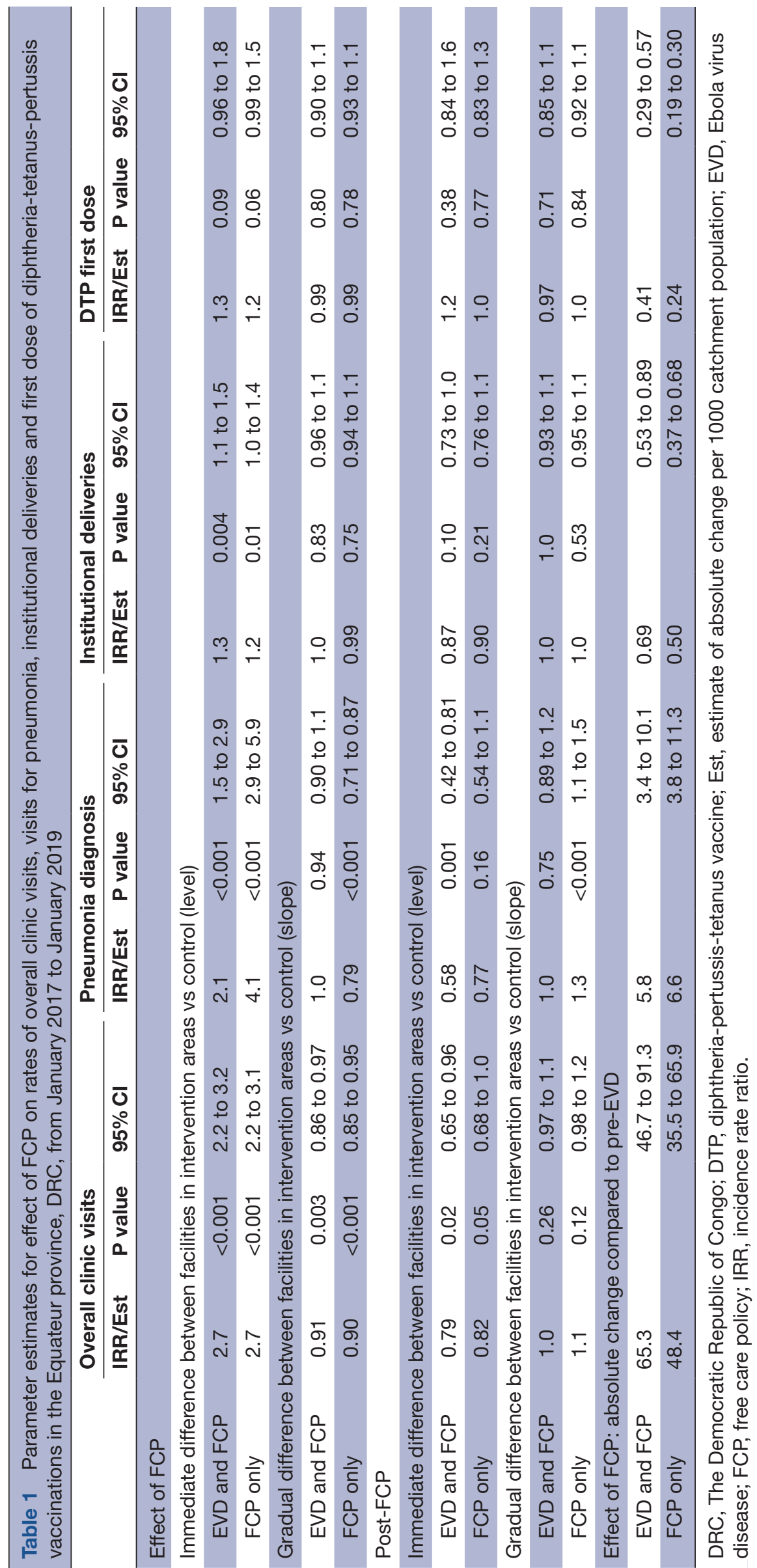




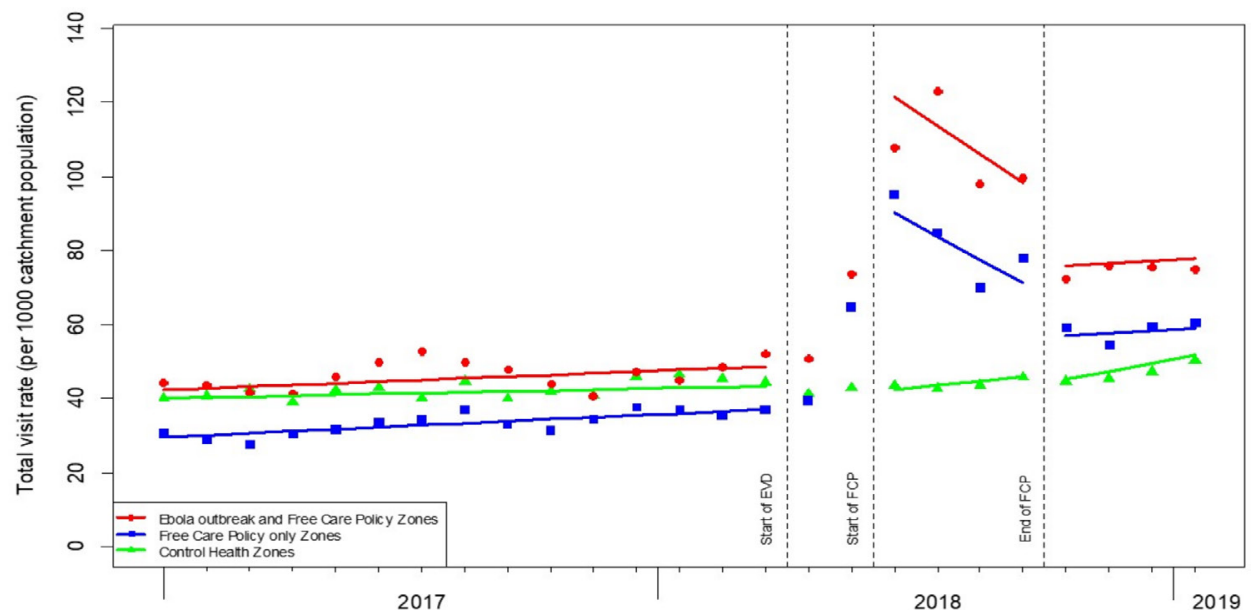

Figure 2 Mean monthly rate of overall clinic visits in health zones by intervention groups and control in Equateur province, January 2017 to January 2019. EVD, Ebola virus disease; FCP, free care policy.

FCP implementation and cessation were similar to pneumonia in the EVD and FCP health zones, while increase in visits for diarrhoea was less drastic than pneumonia in FCP-only health zones. The FCP was associated with an increase in visit rates for malaria in FCP-only health zones (IRR: $1.5,95 \%$ CI: $1.1-2.1, \mathrm{p}=0.02$ ) but not in the EVD and FCP health zones (IRR: 1.1, 95\% CI: 0.69-1.7, $\mathrm{p}=0.72$ ) (see online supplementary appendix).

\section{Maternal health}

The FCP had a comparatively moderate effect on the utilisation of maternal health services both in the EVD and FCP health zones and the FCP-only health zones which varied by service type. Figure 4 shows the model results for institutional deliveries. Compared to control zones, deliveries at clinics increased in all of the FCP health zones immediately following the implementation of the policy (EVD and FCP: IRR: 1.3, 95\% CI: $1.1-1.5, \mathrm{p}=0.004$; FCP-only: IRR: $1.2,95 \%$ CI: 1.1-1.4, $\mathrm{p}=0.01$ ), but the increase did not continue over the whole implementation period (EVD and FCP: IRR: 1.0, 95\% CI: $0.96-1.1, \mathrm{p}=0.83$; FCP only: IRR: 0.99 , $95 \%$ CI: $0.94-1.1, \mathrm{p}=0.75)$. The decreases in the institutional delivery rate at the end of FCP were not statistically significant in EVD and FCP health zones (IRR: 0.87, 95\% CI: $0.73-1.0, \mathrm{p}=0.10)$ nor in FCP-only health zones (IRR: $0.90,95 \%$ CI: $0.76-1.1, \mathrm{p}=0.21$ ), and there was no change in the rate after the policy ended (EVD and FCP: IRR: $1.0,95 \%$ CI: $0.93-1.1, \mathrm{p}=1.0$; FCP only: IRR: 1.0, $95 \%$ CI: $0.95-1.1, p=0.53)$. FCP had a similar effect on first antenatal care visits, but not on fourth antenatal care visits and postnatal care visits (see online supplementary appendix).

\section{Vaccination}

Figure 5 shows the results for the administration of first doses of the DTP vaccine. Reporting of routine immunisation in the EVD and FCP health zones was predominantly from the Wangata health zone. Compared to control health zones, DTP immunisation had no significant change in the EVD and FCP health zones (EVD and FCP: IRR: 1.3, 95\% CI: $0.96-1.8, p=0.09$ ) and marginal increase in FCP-only health zones (IRR: 1.2, 95\% CI: $0.99-1.5, p=0.06$ ). No significant changes were found in the trends nor level after the FCP ended.

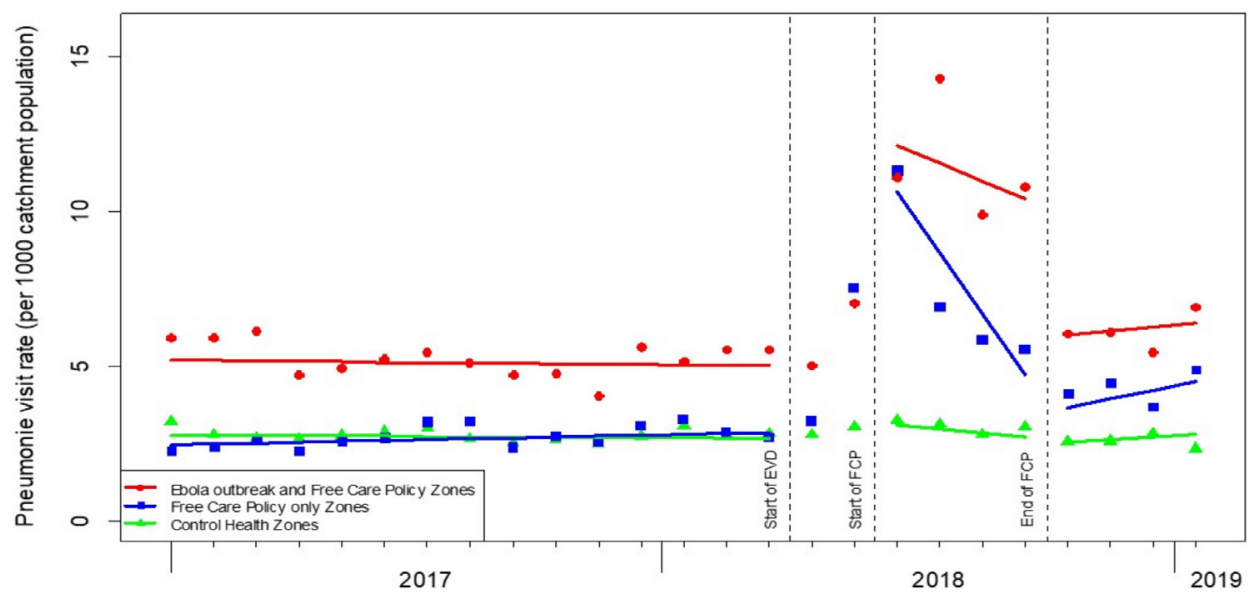

Figure 3 Mean monthly rate of clinic visits for pneumonia in health zones by intervention groups and control in Equateur province, January 2017 to January 2019. EVD, Ebola virus disease; FCP, free care policy. 


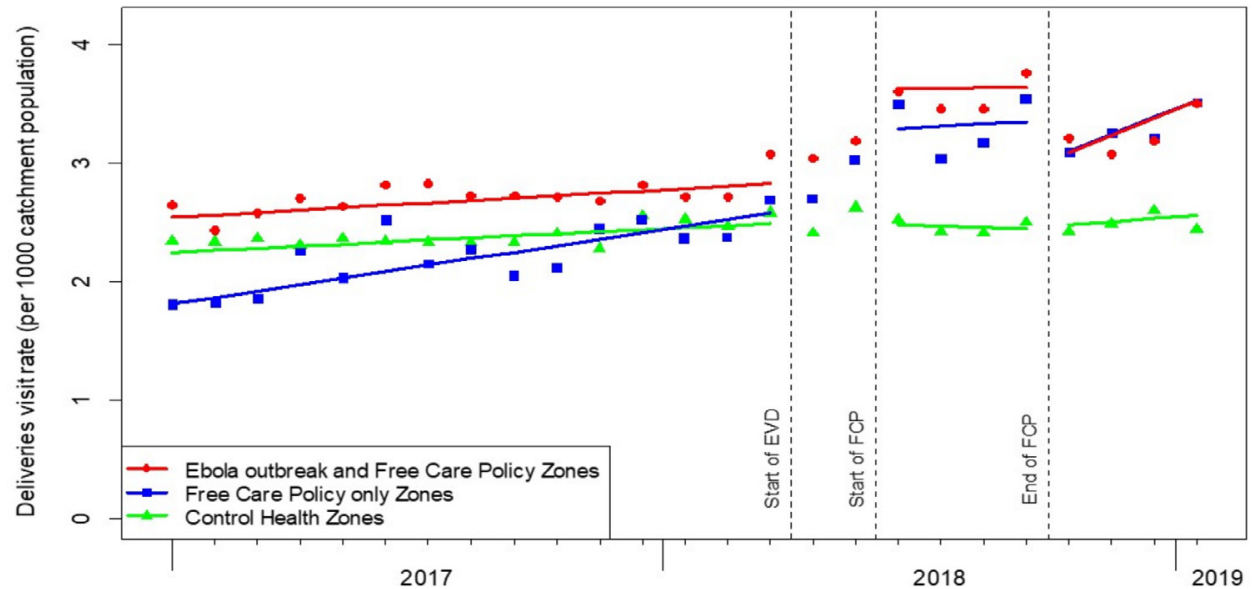

Figure 4 Mean monthly rate of institutional delivery at primary health facilities in health zones by intervention groups and control in Equateur province, January 2017 to January 2019. EVD, Ebola virus disease; FCP, free care policy.

\section{DISCUSSION}

During disease outbreaks, maintaining the use of health services is important both for diagnosing diseases and ensuring continuity of care for other health issues. We found strong evidence that a FCP implemented in the DRC during an ongoing EVD outbreak associated with large increases in the rate of utilisation of health services in PHCs. The magnitude of this increase was similar in the EVD zones compared to neighbouring zones with the FCP but no EVD. Our findings are consistent with previous studies that found short term effects of FCPs in other African contexts, ${ }^{19}{ }^{35}$ but in stark contrast to the finding that fear and a lack of trust greatly curbed health service utilisation in the West African EVD outbreak. ${ }^{679}$

Despite the increase in the use of health services, the FCP was not equally effective for all indicators. The largest increase was observed in curative visits for pneumonia and diarrhoea, while increases in the treatment of malaria were observed only in the FCP-only zones. Preventative services such as first antenatal visit and institutional delivery showed more modest increases. This pattern generally aligns with other FCP studies that have shown greater effectiveness for curative services than preventative services. ${ }^{1516}$ Studies from West Africa also suggest the use of curative services recovered and rebounded earlier in the post-outbreak period, ${ }^{5}$ compared to preventative services. ${ }^{19}$ Due to the concern of EVD transmission, health facilities may have curbed the delivery of services that involved needles or blood extraction. ${ }^{36}$ Indeed, the Chief Medical Officer of Bikoro health zone reported that EVD responders recommended that, during the epidemic, routine immunisation, elective surgeries and malaria rapid diagnostic testing be curbed in the epicentre health zones (Dr B Loleka, oral communication, 8 May 2019). The restriction in routine immunisation in the epicentre may have also contributed to reduced reporting on immunisation and malaria diagnosis during the EVD outbreak. Although these services were not targeted by the FCP, we found some evidence that DTP vaccination and malaria diagnosis did not decrease during the EVD period in the reporting PHCs, suggesting that such activities were maintained in areas outside of the EVD epicentre.

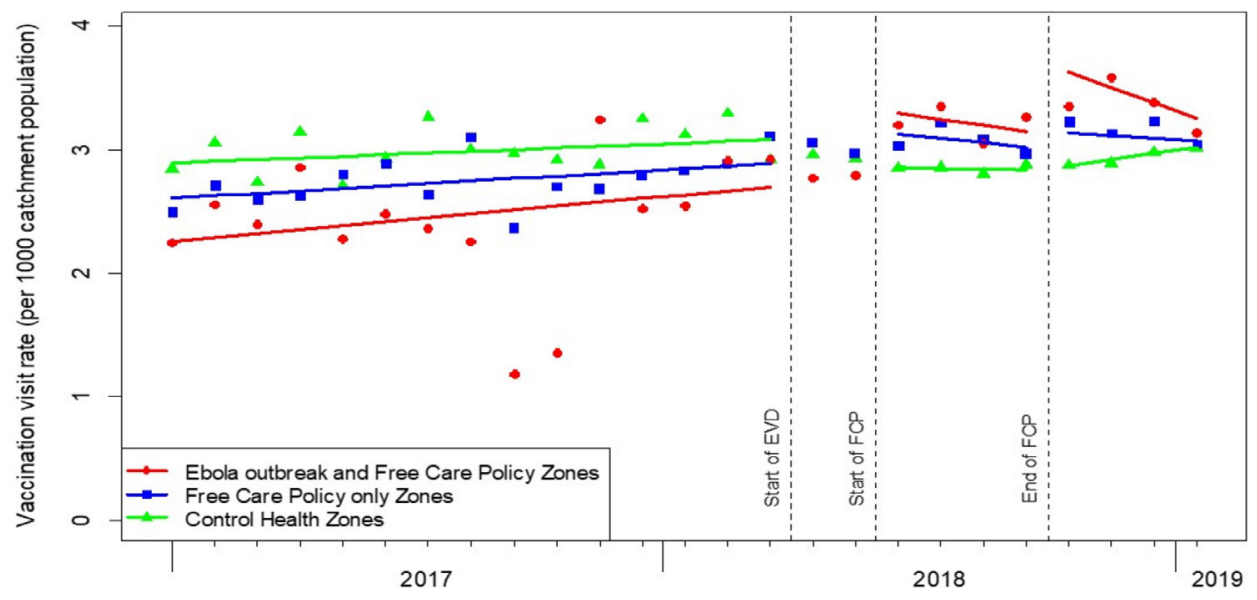

Figure 5 Mean monthly rate of administration of first dose of diphtheria-tetanus-pertussis vaccinations in health zones by intervention groups and control in Equateur province, January 2017 to January 2019. Note: Ebola outbreak and free care policy (FCP) health zones contain health centres from predominantly the Wangata health zone; analysis included no facility from Iboko health zone. EVD, Ebola virus disease. 
Our findings also highlight some potential challenges in implementing FCPs. The rapid increases in the use of services following the implementation of the policy attenuated over the following 3 months which may be a result of the disruption of the FCP on the local health system. ${ }^{37}$ In particular, the sudden increase in demand for primary health services may have overstretched the limited human resources ${ }^{38}$ or disrupted regular operations due to the changes in reimbursements paid to health workers and budgetary constraints during the FCP implementation. ${ }^{39}$ These impacts should be considered in future uses of FCP-type policies designed to mitigate the impact of infectious disease outbreaks.

Our findings also provide some insights that could be useful to decision-makers contemplating setting up similar policies in other infectious disease outbreak contexts, for example, countries currently deciding how to respond to the pandemic of COVID-19. First, we demonstrate that the policy was effective soon after implementation which was likely due in part to the presence of an existing payment structure that had previously been established in the region and that could quickly be leveraged for this programme. Without such a platform, it may be challenging for other countries to implement such a policy in a rapid manner. Second, while the intent had always been for the policy to be temporary, our findings suggests that the effectiveness of the policy began to wane soon after implementation, potentially as a result of the lack of longer-term planning. Decision-makers should try to better balance the need for short-term effectiveness with the sustainability of the policy, in particular when it is uncertain at the onset how long an outbreak will last.

Our study has a number of limitations that should be considered when interpreting our results. First, our sample included only health centres and did not include all health facilities. As health centres are the formal health system structure that provide primary health services, we did not include health posts, which provide mainly community health services and health promotion activities. ${ }^{40}$ Our sample also excluded hospitals and private health facilities. As private facilities are not directly governed by the Ministry of Public Health, their reporting of routine health data is limited. It was not possible for us to include hospitals as their reporting in some health zones was very inconsistent during the outbreak. However, in the Appendix we present data from select hospitals and note that similar increases in use of health services were also observed. Second, there was a small increase in missing data during the first few months of the EVD outbreak, particularly in vaccination and malaria diagnosis. As we excluded health centres with consecutive missing data in this period, our samples for these two indicators did not include all EVD health zones. Hence, our findings on vaccination and visits for malaria diagnosis may not be generalisable to the entire EVD outbreak area. Third, we were unable to include an estimate of the level and trend changes following the Ebola outbreak as there were only 2 months between the outbreak and the start of the FCP. Finally, it is possible that the intervention may have had led to some spillover effects into neighbouring health zones which we are not able to fully control for in our analysis. However, the challenging terrain and large distances to health facilities may have limited spillover effects. Plus, if such spillover effects had happened, it is unclear which direction they would have gone, and could have even made it less likely that we were to find an effect.

\section{CONCLUSION}

In conclusion, our study demonstrates that the introduction of a FCP was strongly associated with rapid increases in the use of health services, in particular in zones with both the policy and EVD. This is in contrast to prior EVD outbreaks, wherein countries did not implement FCPs at scale and saw large declines in the use of health services, suggesting that such policies may be effective at mitigating the impact of future EVD outbreaks. However, the increases were not uniform across all health services and the rapid increases in the use of health services did not continue over the full FCP period. FCPs may be an effective way to mitigate the impact of future outbreaks, including the current pandemic of COVID-19 that is now threatening many countries including the DRC, on population health, however, more research is needed to better understand the impact in different contexts and how such policies can be effective over time.

\section{Author affiliations}

${ }^{1}$ Health Sciences, Wilfrid Laurier University, Waterloo, Ontario, Canada

${ }^{2}$ Centre for Health Services and Policy Research, The University of British Columbia Vancouver, British Columbia, Canada

${ }^{3}$ Independent Consultant, Brookline, Massachusetts, USA

${ }^{4}$ Department of Anthropology, Harvard University, Cambridge, Massachusetts, USA

${ }^{5}$ Bluesquare, Brussels, Belgium

${ }^{6}$ Public Health School of Kinshasa, Université de Kinshasa, Kinshasa, The Democratic Republic of Congo

${ }^{7}$ School of Public Health, University of Rwanda, Kigali, Rwanda

${ }^{8}$ Ministry of Public Health, Kinshasa, The Democratic Republic of Congo

${ }^{9}$ School of Public Health, University of Hong Kong, Pokfulam, Hong Kong SAR

Twitter Michael R Law @myclaw, Sharon Abramowitz @anthromama, Grégoire Lurton@grlurton, Serge Manitu Mayaka @SergeMayaka and Karen A Grépin @KarenGrepin

Acknowledgements We acknowledge that this manuscript was prepared on the Haldimand Tract, traditional territory of the Neutral, Anishinaabe and Haudenosaunee peoples. We are grateful to all of our key informants, including those in the Democratic Republic of Congo (DRC) and elsewhere to helping us obtain information on key parameters related to the outbreak. We also acknowledge Qamar Mahmood and Sofia Rossell at International Development Research Centre for their support, Jess Wilhelm's research assistance on the project, and Nicolas de Borman's facilitation in facilitating the project and data access.

Contributors MRL, SI, SMM and KG conceived the idea. YWH, MRL, SI, LA-S, GL, $\mathrm{SMM}, \mathrm{HR}$ and $\mathrm{KG}$ developed the protocol and contributed to the study design. GL and RO provided study data and assisted with data management. YWH and LC managed and analysed the data, in collaboration with MRL. YWH and KG drafted the manuscript. All authors reviewed the manuscript and contributed to the revision of the manuscript, and approved its final version. The corresponding author had full 
access to all the data in the study and had final responsibility for the decision to submit for publication.

Funding International Development Research Centre, Social Sciences and Humanities Research Council, Canadian Institutes of Health Research, and the AXA Research Fund. This project received funding from the Rapid Research Fund for Ebola supported by the International Development Research Centre, Social Sciences and Humanities Research Council, and Canadian Institutes of Health Research. Dr. Law received salary support through a Canada Research Chair and a Michael Smith Foundation for Health Research Scholar Award. Dr. Grépin also received salary support through a Canada Research Chair

Disclaimer The funders of the study had no role in study design, data collection, data analysis, interpretation of data, or writing of the report.

Map disclaimer The depiction of boundaries on this map does not imply the expression of any opinion whatsoever on the part of BMJ (or any member of its group) concerning the legal status of any country, territory, jurisdiction or area or of its authorities. This map is provided without any warranty of any kind, either express or implied.

Competing interests MRL has consulted for Health Canada, the Health Employees' Union, the Conference Board of Canada, and provided expert witness testimony for the Attorney General of Canada. Bluesquare has ongoing contracts with a variety of organisations in DRC including the Ministry of Health and the World Bank. SI, LA-S, SMM, and HR were paid as individual consultants as part of their collaboration with this project.

Patient and public involvement Patients and/or the public were not involved in the design, or conduct, or reporting, or dissemination plans of this research.

Patient consent for publication Not required.

Provenance and peer review Not commissioned; externally peer reviewed

Data availability statement Data may be obtained from a third party and are not publicly available. Data are obtainable from the Ministry of Public Health in the DRC.

Open access This is an open access article distributed in accordance with the Creative Commons Attribution Non Commercial (CC BY-NC 4.0) license, which permits others to distribute, remix, adapt, build upon this work non-commercially, and license their derivative works on different terms, provided the original work is properly cited, appropriate credit is given, any changes made indicated, and the use is non-commercial. See: http://creativecommons.org/licenses/by-nc/4.0/.

\section{ORCID iDs}

Michael R Law http://orcid.org/0000-0002-5637-60

Sharon Abramowitz http://orcid.org/0000-0003-3671-8842

Lys Alcayna-Stevens http://orcid.org/0000-0003-0653-1884

Grégoire Lurton http://orcid.org/0000-0002-3714-6893

Romain Olekhnovitch http://orcid.org/0000-0003-0457-5904

Gabriel Kyomba http://orcid.org/0000-0003-2695-7487

Hinda Ruton http://orcid.org/0000-0003-0538-275X

Karen A Grépin http://orcid.org/0000-0003-4368-0045

\section{REFERENCES}

1 Wagenaar BH, Augusto O, Beste J, et al. The 2014-2015 Ebola virus disease outbreak and primary healthcare delivery in Liberia: timeseries analyses for 2010-2016. PLoS Med 2018;15:e1002508.

2 Chang $\mathrm{H}-\mathrm{J}$, Huang $\mathrm{N}$, Lee $\mathrm{C}-\mathrm{H}$, et al. The impact of the SARS epidemic on the utilization of medical services: SARS and the fear of SARS. Am J Public Health 2004;94:562-4.

3 Jones SA, Gopalakrishnan S, Ameh CA, et al. 'Women and babies are dying but not of Ebola': the effect of the Ebola virus epidemic on the availability, uptake and outcomes of maternal and newborn health services in Sierra Leone. BMJ Glob Health 2016;1:e000065.

4 McQuilkin PA, Udhayashankar K, Niescierenko M, et al. Health-Care access during the Ebola virus epidemic in Liberia. Am J Trop Med Hyg 2017;97:931-6.

5 Morse B, Grépin KA, Blair RA, et al. Patterns of demand for non-Ebola health services during and after the Ebola outbreak: panel survey evidence from Monrovia, Liberia. BMJ Glob Health 2016;1:e000007.

6 McLean KE, Abramowitz SA, Ball JD, et al. Community-Based reports of morbidity, mortality, and health-seeking behaviours in four Monrovia communities during the West African Ebola epidemic. Glob Public Health 2018;13:528-44.
7 Wilhelm JA, Helleringer S. Utilization of non-Ebola health care services during Ebola outbreaks: a systematic review and metaanalysis. J Glob Health 2019;9:10406.

8 Sochas L, Channon AA, Nam S. Counting indirect crisis-related deaths in the context of a low-resilience health system: the case of maternal and neonatal health during the Ebola epidemic in Sierra Leone. Health Policy Plan 2017;32:iii32-9.

9 Delamou A, Ayadi AME, Sidibe S, et al. Effect of Ebola virus disease on maternal and child health services in guinea: a retrospective observational cohort study. Lancet Glob Health 2017;5:e448-57.

10 Plucinski MM, Guilavogui T, Sidikiba S, et al. Effect of the Ebolavirus-disease epidemic on malaria case management in guinea, 2014: a cross-sectional survey of health facilities. Lancet Infect Dis 2015;15:1017-23.

11 Walker PGT, White MT, Griffin JT, et al. Malaria morbidity and mortality in Ebola-affected countries caused by decreased healthcare capacity, and the potential effect of mitigation strategies: a modelling analysis. Lancet Infect Dis 2015;15:825-32.

12 Tattevin P, Baysah MK, Raguin G, et al. Retention in care for HIV-infected patients in the eye of the Ebola storm: lessons from Monrovia, Liberia. AIDS 2015;29:N1-2.

13 Leuenberger D, Hebelamou J, Strahm S, et al. Impact of the Ebola epidemic on general and HIV care in Macenta, forest guinea, 2014. AIDS 2015;29:1883-7.

14 Takahashi S, Metcalf CJE, Ferrari MJ, et al. Reduced vaccination and the risk of measles and other childhood infections post-Ebola. Science 2015;347:1240-2.

15 Ridde V, Morestin F. A scoping review of the literature on the abolition of user fees in health care services in Africa. Health Policy Plan 2011;26:1-11.

16 Lagarde M, Palmer $\mathrm{N}$. The impact of user fees on access to health services in low- and middle-income countries. Cochrane Database Syst Rev : 2011:CD009094.

17 Obare F, Abuya T, Matanda D, et al. Assessing the communitylevel impact of a decade of user fee policy shifts on health facility deliveries in Kenya, 2003-2014. Int J Equity Health 2018;17:65.

18 Lépine A, Lagarde M, Le Nestour A. How effective and fair is user fee removal? Evidence from Zambia using a pooled synthetic control. Health Econ 2018;27:493-508.

19 Maini R, Van den Bergh R, van Griensven J, et al. Picking up the bill - improving health-care utilisation in the Democratic Republic of Congo through user fee subsidisation: a before and after study. BMC Health Serv Res 2014;14:504.

20 Robert E, Samb OM, Marchal B, et al. Building a middle-range theory of free public healthcare seeking in sub-Saharan Africa: a realist review. Health Policy Plan : 2017;32:1002-1014.

21 Ministre de la santé de la RDC, World Health Organization Regional Office for Africa. Brève situation de la Riposte l'épidémie de la Maladie Virus Ebola (MVE) dans la Province de l'Equateur, République Démocratique du Congo au 30e jour [Brief Situation of Ebola Virus Disease (EVD) Response in Equateur Province, Democratic Republic of C. Kinshasa, Democratic Republic of Congo, 2018. Available: https://www.afro.who.int/publications/ breve-situation-de-la-riposte-lepidemie-de-la-maladie-virus-ebolamve-dans-la-province

22 Ministère du Plan et Suivi de la Mise en oeuvre de la Révolution de la Modernité. République Démocratique du Congo. Enquête Démographique et de Santé (EDS-RDC) 2013-2014 [Demographic and Health Survey 2013-2014]. Kinshasa, 2014.

23 Ministere de la Sante Publique. Repubique Democratique Du Congo Ministere De La Sante Publique Plan National De Development Sanitaire 2016-2020: vers la couverture sanitaire universelle [Democratic Republic of the Congo Ministry of Public Health National Development Plan 2016-2020: towa. Kinshasa, Democratic Republic of Congo, 2016.

24 Laokri S, Soelaeman R, Hotchkiss DR. Assessing out-of-pocket expenditures for primary health care: how responsive is the Democratic Republic of Congo health system to providing financial risk protection? BMC Health Serv Res 2018;18:1-19.

25 Ilunga Kalenga O, Moeti M, Sparrow A, et al. The ongoing Ebola epidemic in the Democratic Republic of Congo, 2018-2019. N Engl J Med 2019;381:373-383

26 Barry A, Ahuka-Mundeke S, Ali Ahmed Y, et al. Outbreak of Ebola virus disease in the Democratic Republic of the Congo, April-May, 2018: an epidemiological study. The Lancet 2018;392:213-21.

27 World Health Organization. Ebola virus disease Democratic Republic of the Congo: external situation report 2, 2018.

28 World Health Organization. Ebola virus disease Democratic Republic of the Congo: external situation report 17, 2018. 
29 Wagner AK, Soumerai SB, Zhang F, et al. Segmented regression analysis of interrupted time series studies in medication use research. J Clin Pharm Ther 2002;27:299-309.

30 Measure Evaluation. Mapping the Stages of MEASURE Evaluation's Data Use Continuum to DHIS 2: An Example from the Democratic Republic of the Congo. Chapel Hill, USA, 2019.

31 Yourkavitch J, Prosnitz D, Herrera S. Data quality assessments stimulate improvements to health management information systems: evidence from five African countries. J Glob Health 2019;9:10806.

32 Ruton $\mathrm{H}$, Musabyimana A, Gaju E, et al. The impact of an mHealth monitoring system on health care utilization by mothers and children: an evaluation using routine health information in Rwanda. Health Policy Plan 2018;33:920-7.

33 Ngabo F, Tate JE, Gatera M, et al. Effect of pentavalent rotavirus vaccine introduction on hospital admissions for diarrhoea and rotavirus in children in Rwanda: a time-series analysis. Lancet Glob Health 2016;4:e129-36.

34 SAS Institute Inc. Sas 9.4 statements: reference, 2013.

35 Zombré D, De Allegri M, Ridde V. Immediate and sustained effects of user fee exemption on healthcare utilization among children under five in Burkina Faso: a controlled interrupted time-series analysis. Soc Sci Med 2017;179:27-35.

36 Elston JWT, Moosa AJ, Moses F, et al. Impact of the Ebola outbreak on health systems and population health in Sierra Leone. J Public Health 2016;38:673-8.

37 Ridde V, Robert E, Meessen B. A literature review of the disruptive effects of user fee exemption policies on health systems. BMC Public Health 2012;12:289.

38 Witter S, Wurie H, Bertone MP. The free health care initiative: how has it affected health workers in Sierra Leone? Health Policy Plan 2016;31:1-9.

39 Alcayna-Stevens L. Planning for Post-Ebola: Lessons Learned from DR Congo's 9th Epidemic. New York, 2018.

40 Ecole de Sante Publique de Kinshasa (ESPK). Republique Democratique du Congo Evaluation des Presentations des Services de souns de Sante EPSS 2017-2018 Rapport Final [Democratic Rebublic of Congo Evaluation of Health Services EPSS 2017-2018 Final report], 2019. Available: http://dhsprogram. com/pubs/pdf/SPA30/SPA30.pdf 